\title{
Principal Component Analysis In Radar Polarimetry
}

\author{
A. Danklmayer ${ }^{1}$, M. Chandra ${ }^{2}$, and E. Lüneburg ${ }^{3}$ \\ ${ }^{1}$ Institut für Hochfrequenztechnik und Radartechnik, Deutsches Zentrum für Luft- und Raumfahrt, P.O. Box 1116, 82234 \\ Weßling, Germany \\ ${ }^{2}$ Professur für Hochfrequenztechnik und Photonik, Technische Universität Chemnitz, Reichenhainer Str. 70, 09126 \\ Chemnitz, Germany \\ ${ }^{3}$ Ernst Lüneburg, EML-Consultants, Georg-Schmied-Weg 04, 82234 Oberpfaffenhofen, Germany
}

\begin{abstract}
Second order moments of multivariate (often Gaussian) joint probability density functions can be described by the covariance or normalised correlation matrices or by the Kennaugh matrix (Kronecker matrix). In Radar Polarimetry the application of the covariance matrix is known as target decomposition theory, which is a special application of the extremely versatile Principle Component Analysis (PCA). The basic idea of PCA is to convert a data set, consisting of correlated random variables into a new set of uncorrelated variables and order the new variables according to the value of their variances. It is important to stress that uncorrelatedness does not necessarily mean independent which is used in the much stronger concept of Independent Component Analysis (ICA). Both concepts agree for multivariate Gaussian distribution functions, representing the most random and least structured distribution.

In this contribution, we propose a new approach in applying the concept of PCA to Radar Polarimetry. Therefore, new uncorrelated random variables will be introduced by means of linear transformations with well determined loading coefficients. This in turn, will allow the decomposition of the original random backscattering target variables into three point targets with new random uncorrelated variables whose variances agree with the eigenvalues of the covariance matrix. This allows a new interpretation of existing decomposition theorems.
\end{abstract}

\section{Introduction}

In radar and optical polarimetry there exists essentially two different methods to characterize polarimetric scattering properties of plane electromagnetic waves scattered by randomly distributed targets using second order multivariate statistics (Boerner et al., 1998; Mott, 1992; Krogager, 1993; Lüneburg, 1995). The first is the Kennaugh matrix which is used for finding solutions for maximal and minimal power transfer between transmitting and receiving antennas. The

Correspondence to: A. Danklmayer

(andreas.danklmayer@dlr.de) second is the covariance matrix where the analysis is used for entropy and variance considerations and for the generation of uncorrelated random variables.

The basic problem of principal component analysis (Jollife, 2002) is how to find a suitable representation of multivariate data in order to make the essential structure more visible and to identify any distinct feature. The main purpose of PCA is to convert a set of possibly correlated random variables into new uncorrelated variables. For existing decomposition theorems cf. Cloude and Pottier (1996).

\section{Principle Component Analysis (PCA)}

With Principle Component Analyis one tries to find new variables which are uncorrelated but not necessary independent. As a starting point the scattering matrix represented by the $2 \times 2 S$-Matrix is considered, describing completely the polarization transforming properties of a target at a single frequency in the reference direction.

$S=\left(\begin{array}{ll}S_{H H} & S_{H V} \\ S_{V H} & S_{V V}\end{array}\right)$

The indices of the matrix elements represent the transmit and receive polarization of the plane electromagnetic wave.

The vec operation (Horn and Johnson, 1985) is used to arrive at the target feature vector $\boldsymbol{k}$ given by

$\boldsymbol{k}_{4}(t)=\operatorname{vec} S(t)=\left[\begin{array}{c}S_{H H}(t) \\ S_{V H}(t) \\ S_{H V}(t) \\ S_{V V}(t)\end{array}\right]$,

where the vec operation can be considered as a simple stacking of the columns of the scattering matrix $S$.

This target feature vector is used to calculate the $4 \times 4$ covariance matrix explicitly according to

$C_{4}=<\boldsymbol{k}_{4}(t) \boldsymbol{k}_{4}^{\dagger}(t)>$

where the dagger symbol represents complex conjugation and transposition. 
$C_{4}=\left[\begin{array}{cccc}<\left|S_{H H}(t)\right|^{2}> & <S_{H H}(t) S_{V H}^{*}(t)> & <S_{H H}(t) S_{H V}^{*}(t)> & <S_{H H}(t) S_{V V}^{*}(t)> \\ <S_{V H}(t) S_{H H}^{*}(t)> & <\left|S_{V H}(t)\right|^{2} & <S_{V H}(t) S_{H V}^{*}(t)> & <S_{V H}(t) S_{V V}^{*}(t)> \\ <S_{H V}(t) S_{H H}^{*}(t)> & <S_{H V}(t) S_{V H}^{*}(t)> & <\left|S_{H V}(t)\right|^{2}> & <S_{H V}(t) S_{V V}^{*}(t)> \\ <S_{V V}(t) S_{H H}^{*}(t)> & <S_{V V}(t) S_{V H}^{*}(t)> & <S_{V V}(t) S_{H V}^{*}(t)> & <\left|S_{V V}(t)\right|^{2}>\end{array}\right]$

Being Hermitian positive semidefinite the covariance matrix $C_{4}$ can be unitarily diagonalized.

$U^{-1} C_{4} U=\Lambda \equiv \operatorname{diag}\left[\lambda_{1}, \lambda_{2}, \lambda_{3}, \lambda_{4}\right]$

with $U=\left[\hat{\boldsymbol{x}}_{1}, \hat{\boldsymbol{x}}_{2}, \hat{\boldsymbol{x}}_{3}, \hat{\boldsymbol{x}}_{4}\right]$.

$\lambda_{i}(i=1, \ldots 4)$ are denoting the eigenvalues of the covariance matrix and $\hat{\boldsymbol{x}}_{i}(i=1, \ldots 4)$ are the eigenvectors, respectively.

Now we introduce the new target feature vector $\boldsymbol{Z}(t)$ by a linear transformation

$\boldsymbol{Z}(t)=\left[\begin{array}{c}z_{1} \\ z_{2} \\ z_{3} \\ z_{4}\end{array}\right]=U^{\dagger} \boldsymbol{k}(t)=\left[\begin{array}{c}x_{1}^{\dagger} k(t) \\ x_{2}^{\dagger} k(t) \\ x_{3}^{\dagger} k(t) \\ x_{4}^{\dagger} k(t)\end{array}\right]$ or $\boldsymbol{k}(t)=U \cdot \boldsymbol{Z}(t)(6)$

The components $z_{i}(i=1, \ldots 4)$ are called the principle components. Furthermore the spectral decomposition of the covariance matrix is given by

$C_{4}=U \Lambda U^{\dagger}=\sum_{i=1}^{4} \lambda_{i} x_{i} x_{i}+^{\dagger}=\sum_{i=1}^{4} \lambda_{i} C_{4, i}$

where $C_{4 i}$ are $4 \times 4$ covariance matrices of point targets with rank 1. The reverse vec operation may be applied to $x i$ $(\mathrm{i}=1 . .4)$ and the results can be interpreted as $2 \times 2$ elementary deterministic point targets $S_{i}$ with $\operatorname{span}\left(S_{i}\right)=1$

$\boldsymbol{x}_{\boldsymbol{i}}=$ vec $S_{i}=\left[\begin{array}{l}x_{i, 1} \\ x_{i, 2} \\ x_{i, 3} \\ x_{i, 4}\end{array}\right] \Leftrightarrow S_{i}=\left[\begin{array}{ll}x_{i, 1} & x_{i, 3} \\ x_{i, 2} & x_{i, 4}\end{array}\right](\mathrm{i}=1, \ldots, 4)$

and hence using the relation $\boldsymbol{k}(t)=U \boldsymbol{Z}(t)$

$S(t)=\left[\begin{array}{ll}S_{x x} & S_{x y} \\ S_{y x} & S_{y y}\end{array}\right]=\sum_{i=1}^{4} z_{i}(t)\left[\begin{array}{ll}x_{i 1} & x_{i 3} \\ x_{i 2} & x_{i 4}\end{array}\right]=\sum_{i=1}^{4} z_{i}(t) S_{i}$

\section{Conclusion}

Having a series of coherent observations given by the scattering matrix $S$ the principle component analysis provides a link to the incoherent method of interpretation (covariance matrix analysis) and furthermore an equivalent coherent representation containing a maximum of 4 possible uncorrelated features. The target description can be formed using $z_{i}$ values and $S_{i}$ point scatter matrices. This contribution provides some theory which is necessary for the application of PCA in radar polarimetry. Further work will include the analysis of different types of scatterers according to the proposed method and an intercomparison with other methods (Alberga, 2004).

Acknowledgements. The authors wish to thank the EU for supporting this research in the frame of the Research Training Network (RTN)-AMPER. Contract number: HPRN-CT-2002-00205.

\section{References}

Alberga, V.: Comparison of polarimetric methods in image classification and SAR interferometry applications, $\mathrm{PhD}$ thesis, Tech. Univ. Chemnitz, Chemnitz, Germany, Available: http://archiv. tu-chemnitz.de/pub/2004/0125, 2004.

Boerner, W.-M., Mott, H., Lüneburg, E., Brisco, B., Brown, R., and Paterson, J. S. (authors); Cloude, S. R., Krogager, E., Lee, J. S., Schuler, D. L., van Zyl, J. J., Randall, D., and Budkewitsch, P. (contributing authors): Polarimetry in Remote Sensing: Basic and Applied Concepts, Chapter 5, in: The Manual of Remote Sensing, edited by: Reyerson, R. A., 3rd Edition (American Society of Photogrammetry and Remote Sensing, ASPRS Publishing, Bethesda, MD 1998), 1998.

Cloude, S. R. and Pottier, E.: A review of target decomposition theorems in radar polarimetry, IEEE Trans. GRS, vol. 34(2), 498518, Mar. 1996.

Horn, R. A. and Johnson, Ch. R.: Matrix Analysis, Cambridge University Press, New York, 1985.

Jolliffe, I. T.: Principal Component Analysis, 2nd edn., Springer New York 2002.

Krogager, E.: Aspects of Polarimetric Radar Imaging, PhD thesis, Technical University of Denmark, 1993.

Lüneburg, E.: IEICETransactions on Electronics (Special Issue on Electromagnetic Theory), E78-C 10, 1339(1995), 1995.

Mott, H.: Antennas for Radar and Communications, A Polarimetric Approach, John Wiley and Sons, New York, 1992. 